\title{
Características sobre o uso e abuso de drogas, alterações cognitivas e desempenho ocupacional de usuários assistidos pelo CAPS AD
}

\author{
Characteristics of drug use and abuse, cognitive impairments and occupational performance of \\ users assisted by Psychosocial Care Center of Alcohol and other drugs \\ Características sobre el uso y abuso de drogas, cambios desempeño cognitivo y ocupacional de \\ usuarios asistidos por CAPS AD
}

Recebido: 20/07/2021 | Revisado: 26/07/2021 | Aceito: 04/08/2021 | Publicado: 09/08/2021

\author{
Maíra Rodrigues dos Santos \\ ORCID: https://orcid.org/0000-0001-8793-0400 \\ Universidade Federal de Pernambuco, Brasil \\ E-mail: mairarodrigues.to@gmail.com \\ Marina Araújo Rosas \\ ORCID: https://orcid.org/0000-0002-5666-7133 \\ Universidade Federal de Pernambuco, Brasil \\ E-mail: marina.rosas@ufpe.br \\ Luziana Carvalho de Albuquerque Maranhão \\ ORCID: https://orcid.org/0000-0002-8456-9135 \\ Universidade Federal de Pernambuco, Brasil \\ E-mail: luziana.maranhao@ufpe.br \\ Ada Salvetti Cavalcanti Caldas \\ ORCID: https://orcid.org/0000-0002-6862-3728 \\ Instituição: Universidade de Pernambuco, Brasil \\ E-mail: adasc@hotmail.com \\ Silvia de Oliveira Luna \\ ORCID: https://orcid.org/0000-0002-7445-0907 \\ Instituição: Prefeitura da cidade do Recife, Brasil \\ E-mail: silviaoluna@yahoo.com.br \\ Maria Gisele Cavalcanti de Oliveira \\ ORCID: https://orcid.org/0000-0001-5096-0075 \\ Universidade Federal de Pernambuco, Brasil \\ E-mail: giselecavalcanti1@hotmail.com \\ Manuela Martins da Silva \\ ORCID: https://orcid.org/0000-0003-2022-8347 \\ Universidade Federal de Pernambuco, Brasil \\ E-mail: manuelamartins30@ outlook.com \\ Mariana Barboza Ferreira \\ ORCID: https://orcid.org/0000-0001-5046-3458 \\ Universidade Federal de Pernambuco, Brasil \\ E-mail: mbf6@outlook.com \\ Vera Lucia Dutra Facundes \\ ORCID: https://orcid.org/0000-0002-4188-7475 \\ Universidade Federal de Pernambuco, Brasil \\ E-mail: vera.facundes@ufpe.br
}

\begin{abstract}
Resumo
Introdução: O uso e abuso de substâncias psicoativas, como o álcool e outras drogas, têm crescido através dos anos no Brasil. O uso abusivo dessas substâncias pode resultar em diversas alterações, incluindo alterações cognitivas, que podem impactar negativamente o desempenho ocupacional das atividades cotidianas. Objetivo: Descrever o perfil de usuários do Centro de Atenção Psicossocial Álcool e Drogas (CAPS AD) e verificar como o uso abusivo de substâncias pode afetar componentes cognitivos e a execução de atividades significativas desses sujeitos. Método: Estudo transversal e descritivo, de abordagem quantitativa, com 45 sujeitos em tratamento no CAPS AD de um município de Pernambuco. Utilizou-se um questionário elaborado pelos pesquisadores, o Montreal Cognitive Assessment (MoCA) e a Medida Canadense de Desempenho Ocupacional (COPM) para a coleta de dados. Resultados: Os resultados revelaram a maioria de usuários masculinos, com baixa escolaridade e início de uso de substâncias ainda na adolescência. Identificou-se 93,3\% de sujeitos com comprometimento cognitivo, principalmente nas funções da linguagem, função executiva, habilidades visuo-espaciais e memória de curto prazo. As principais atividades apontadas como prejudicadas foram trabalhar, se relacionar com a família e estudar. O estudo apontou que
\end{abstract}


houve associação estatisticamente significativa entre as variáveis escolaridade, frequência do uso de drogas, em relação ao comprometimento cognitivo. Conclusão: Os participantes avaliados mostraram prejuízos na cognição e no desempenho de atividades significativas para os mesmos e identificar tais prejuízos pode ajudar no planejamento do tratamento, a fim de melhorar o desempenho cognitivo e ocupacional, e a qualidade de vida dos usuários.

Palavras-chave: Análise e desempenho de tarefas; Cognição; Drogas; Serviços de saúde mental.

\begin{abstract}
Introduction: The use and abuse of psychoactive substances, such as alcohol and other drugs, have grown over the years in Brazil. The abusive use of these substances can result in several alterations, including cognitive impairments that can negatively impact occupational performance of activities of daily life. Objective: To describe the profile of users of the Center for Psychosocial Care Centers Alcohol and others Drugs (CAPS AD) and to verify how abusive use of substances can affect cognitive components and the execution of significant activities of these subjects. Method: A cross-sectional and descriptive study, with a quantitative approach, with 45 subjects under treatment in the CAPS AD of a city of Pernambuco. A questionnaire developed by the researchers, the Montreal Cognitive Assessment (MoCA) and the Canadian Occupational Performance Measure (COPM) was used to data collection. Results: The results revealed the majority of male users, with low level of education and substance use beginning in adolescence. It was identified $93.3 \%$ of subjects with cognitive impairment, especially in the functions of language, executive function, visuospatial skill and short-term memory. The main activities identified as impaired were to work, to relate with family and to study. The study pointed out that there was a statistically significant association between the variables education level, frequency of drug use, and cognitive impairment. Conclusion: Participants showed cognitive impairments and performance impairments of significant activities for them; identify such impairments may help in treatment planning, in order to improve cognitive and occupational performance, and quality of life of individuals.
\end{abstract}

Keywords: Task performance and analysis; Cognition; Drugs; Substance abuse treatment centers.

\title{
Resumen
}

Introducción: El uso y abuso de sustancias psicoactivas, como el alcohol y otras drogas, ha crecido a lo largo de los años en Brasil. El abuso de estas sustancias puede resultar en varios cambios, incluidos cambios cognitivos, que pueden afectar negativamente el desempeño ocupacional de las actividades diarias. Objetivo: Describir el perfil de usuarios del Centro de Atención Psicosocial de Alcohol y Drogas (CAPS AD) y verificar cómo el abuso de sustancias puede afectar los componentes cognitivos de estos sujetos y el desempeño de actividades significativas. Método: Estudio descriptivo transversal, con enfoque cuantitativo, con 45 sujetos en tratamiento en el CAPS AD de un municipio de Pernambuco. Para la recopilación de datos se utilizó un cuestionario desarrollado por los investigadores, la Evaluación Cognitiva de Montreal (MoCA) y la Medida de Desempeño Ocupacional de Canadá (COPM). Resultados: Los resultados revelaron la mayoría de usuarios masculinos, con baja escolaridad y comenzando a consumir sustancias aún en la adolescencia. Se identificó al 93,3\% de los sujetos con deterioro cognitivo, principalmente en funciones del lenguaje, función ejecutiva, habilidades visoespaciales y memoria a corto plazo. Las principales actividades identificadas como discapacitadas fueron el trabajo, la relación con la familia y el estudio. El estudio mostró que existía una asociación estadísticamente significativa entre las variables educación, frecuencia de consumo de drogas, en relación con el deterioro cognitivo. Conclusión: Los participantes evaluados mostraron deficiencias en la cognición y en el desempeño de actividades significativas para ellos; La identificación de tales deficiencias puede ayudar en la planificación del tratamiento, con el fin de mejorar el desempeño cognitivo y ocupacional, y la calidad de vida de los usuarios.

Palabras clave: Análisis de tareas y desempeño; Cognición; Drogas; Servicios de salud mental.

\section{Introdução}

A cultura, o acesso às políticas públicas e o desenvolvimento econômico são fatores que estão ligados às direções históricas do consumo de drogas. Estudos epidemiológicos realizados no Brasil e no mundo mostram que o consumo de substâncias lícitas e ilícitas é considerado um problema político. Devido à complexidade de tal problema, são necessárias políticas governamentais voltadas às ações nas áreas da saúde, educação, justiça, direitos sociais, trabalho, entre outras (Brasil, 2003; Assunção et al, 2019; Vargas; Campos, 2019; Jafelice; Ziliotto; Marcolan, 2020).

No Brasil, segundo o levantamento da Organização Mundial da Saúde (OMS) (2018) o consumo de álcool da população acima de 15 anos, que se refere a 43\% da população, foi de 6,4 litros per capita no ano de 2018. De acordo com o II Levantamento Nacional de Álcool e Drogas (LENAD) realizado no ano de 2012, houve um aumento da população não abstinente de álcool (50\%) comparado com o levantamento no ano de 2006 (48\%). Em relação ao consumo de cocaína e crack 
o LENAD aponta o Brasil como uma das nações emergentes onde o consumo está aumentando, enquanto na maioria dos países está diminuindo. O levantamento também aponta que $6,8 \%$ da população adulta investigada utilizaram a maconha pelo menos uma vez na vida, assim como 3,8\% utilizaram cocaína e 1,3\% fizeram uso do crack (Laranjeira et al, 2014).

Atualmente, o critério de diagnóstico elaborado pela OMS, a Classificação Internacional de Doenças (CID-10), é adotado no Brasil pelo Sistema Único de Saúde (SUS) para ajudar o profissional de saúde a classificar a doença em questão. Há na CID-10 a classificação de "Transtornos Mentais e de Comportamento" que inclui os transtornos provocados pelo uso de substâncias. É possível no diagnóstico indicar o tipo de transtorno e que tipo de substância psicoativa o transtorno está associado, classificando os usuários com "uso nocivo" (ou prejudicial) ou "dependência" de substâncias (Supera, 2017).

De acordo com a OMS (2014), o uso prejudicial de substâncias é um dos principais fatores mundiais para a morbidade, mortalidade e incapacidade dos usuários. As consequências do uso crônico podem resultar em distúrbios de ordem biopsicossocial, lesões contra si mesmo ou aos outros, podendo produzir diversos tipos de alterações no comportamento, percepção, cognição e humor, não tendo um público determinado, discriminação de gênero, raça ou território (Cavalcanti et al, 2020).

A exposição prolongada ao álcool e outras drogas pode causar danos anatômicos no cérebro de um usuário crônico, com um alto nível de perda de neurônios no córtex pré-frontal, desempenhando um importante papel nas alterações cognitivas. Podem-se observar prejuízos significativos na memória, atenção e nas funções executivas como tomada de decisões, resolução de problemas, regulação do comportamento, formação de conceito, planejamento, organização, entre outros (Andrade et al, 2018; Schilindwein-Zanini, R. Sotili, 2019).

A fim de nortear as ações de enfrentamento dos problemas de saúde decorrentes do uso de álcool e outras drogas, o Ministério da Saúde (MS) lançou a Política para Atenção Integral aos usuários de álcool e outras drogas, visando garantir seus direitos assistenciais e assumir de modo integral e articulado o desafio de prevenir, tratar e reabilitar os usuários de álcool e outras drogas (Brasil, 2003; Silva; Abrahão, 2019).

Em 2011 o MS instituiu a Rede de Atenção Psicossocial (RAPS) no âmbito do Sistema Único de Saúde (SUS). Nesta rede de atendimento os Centros de Atenção Psicossocial Álcool e Drogas (CAPS AD) se destacam como serviços de saúde de caráter aberto e comunitário, que atendem pessoas de todas as faixas etárias que apresentam intenso sofrimento psíquico decorrente do uso de crack, álcool e outras drogas (Brasil, 2011).

A equipe que compõe o CAPS AD é multidisciplinar, visto que reúne diversas áreas de atuação. Entre elas está o terapeuta ocupacional que, como parte integrante da equipe do CAPS AD, tem uma atuação que pode contemplar ações de prevenção, assistência direta aos pacientes com sofrimento psíquico, assistência às suas famílias e articulação com outros equipamentos do território, diferindo dos demais profissionais nos objetivos de suas atividades. Os principais objetivos das intervenções realizadas por esses profissionais são resgatar valores pessoais e sociais e ressignificar as atividades cotidianas, tanto as Atividades de Vida Diária (AVD), como as Atividades Instrumentais de Vida Diária (AIVD) (Guariento, 2019; Moares et al, 2020; Machado et al, 2021). As AVD são direcionadas para o autocuidado do sujeito como tomar banho, realizar sua higiene pessoal e alimentar-se. Já as AIVD têm seu foco no suporte dentro de casa ou na comunidade, como gerenciamento do lar e fazer compras, que geralmente são de maior complexidade que as AVD (AOTA, 2020, p.30).

A Estrutura da Prática da Terapia Ocupacional (2020, p.22) define o desempenho ocupacional como "ato de realizar e completar uma ação selecionada (componente de desempenho), atividade ou ocupação, que é resultado da transação dinâmica entre o cliente, o contexto e a atividade". Segundo a Associação Americana de Terapia Ocupacional (AOTA, 2013), o funcionamento do desempenho dos componentes cognitivos está sempre vinculado ao desempenho ocupacional e não pode ser entendido isoladamente. Alterações nesses componentes podem levar a comprometimentos nas AVD, AIVD e atividades de lazer. Portanto, os déficits cognitivos decorrentes do uso abusivo do álcool, crack e outras drogas têm potencial de impactar 
negativamente no desempenho ocupacional do indivíduo, sendo assim necessário compreender a dimensão desse impacto na vida dos usuários (Menezes; Pereira, 2019).

Considerando que a dependência química vem se tornando cada vez mais comum em indivíduos que estão no período da adolescência e se prolongando durante o estágio adulto, identificar os aspectos de fragilidade que podem interferir no desempenho ocupacional desses sujeitos pode contribuir no aperfeiçoamento das práticas de intervenção e cuidados oferecidos pela Terapia Ocupacional aos mesmos.

Sendo assim, o presente estudo tem como objetivo descrever o perfil de usuários do Centro de Atenção Psicossocial Álcool e Drogas e verificar como o uso abusivo de substâncias pode afetar componentes cognitivos e a execução de atividades significativas desses sujeitos.

\section{Metodologia}

Trata-se de um estudo transversal e descritivo, de abordagem quantitativa, com usuários na faixa etária de 19 anos ou mais, em atendimento em dois dos seis CAPS AD da rede de atenção psicossocial de um município no estado de Pernambuco. Foram incluídos usuários com educação formal igual ou maior que quatro anos, considerando 11 anos de escolaridade até a conclusão do ensino médio, em tratamento nos CAPS AD por um período igual ou maior que um mês, considerando o tempo de vinculação com o serviço, que apresentaram uso prejudicial ou dependência de álcool ou outras drogas, conforme os critérios de admissão ao CAPS e estar em acompanhamento dos grupos de tratamento intensivos do CAPS, levando em consideração a frequência dos usuários e o tempo disponível para a coleta de dados. Foram excluídos aqueles que apresentaram como comorbidade alguma doença neurológica, doença psiquiátrica ou outra doença que comprometa a capacidade cognitiva, que não tenham sido decorrentes do uso de substâncias, além dos que não se apresentaram conscientes, orientados e capazes de se expressar oralmente ou com algum comprometimento físico que impossibilitasse a aplicação dos instrumentos. As informações foram previamente colhidas conforme descritas nos prontuários e no momento da entrevista.

No levantamento de dados, baseado nos prontuários disponíveis, realizado entre os meses de novembro e dezembro de 2016, foram identificados 131 usuários nos serviços. Depois de aplicados os critérios de inclusão e exclusão, 32 usuários foram excluídos do estudo, de modo que 99 usuários foram classificados como elegíveis para a pesquisa.

Utilizaram-se três instrumentos para a coleta dos dados, a saber: um questionário elaborado pelos pesquisadores, para coletar os dados sóciodemográfricos (idade, escolaridade, renda, entre outros) e características específicas sobre o uso de substâncias, incluindo perfil do uso/abuso de substâncias e rotina de vida.

Em seguida foi aplicado o Montreal Cognitive Assessment (MoCA), que consiste em um instrumento de rastreio com alta sensibilidade e especificidade para identificar Comprometimentos Cognitivos Leves (CCL), sendo desenvolvido para aqueles sujeitos com demência e comprometimentos cognitivos leves, mas passou a ser utilizado por outras populações. Diversos países realizaram estudos comprovando a eficácia do MoCA com a população de usuários de álcool e outras drogas (Maio et al, 2010; Pedrero-Pérez et al, 2014; Pelletier et al, 2016). A duração de aplicação é aproximada de 10-15 minutos, e através de 12 itens abrange 8 domínios cognitivos (atenção, concentração, funções executivas, memória de trabalho, memória de curto prazo, linguagem, habilidades visuo-espacial, e orientação). $\mathrm{O}$ teste tem como pontuação máxima 30 pontos, onde um escore acima de 26 pontos é considerado sem evidências de prejuízos cognitivos e escores iguais ou menores de 25 pontos são considerados com indicativo de Comprometimento Cognitivo Leve. Deve-se adicionar um ponto para sujeitos com 12 anos ou menos de educação formal. Foi utilizada a versão original validada em português (disponível em: http://www.mocatest.org/wpcontent/uploads/2015/tests-instructions/MoCA-Test-Portuguese_Brazil.pdf) (Nasreddine et al, 2005; Memória et al, 2013).

Com a finalidade de avaliar o desempenho ocupacional foi utilizada a versão brasileira validada da Medida Canadense de Desempenho Ocupacional (COPM). O instrumento, construído para a prática terapêutica ocupacional, visa na primeira 
etapa identificar e na segunda etapa reavaliar por meio de entrevista semiestruturada atividades que, dentro do contexto do adoecimento, o sujeito julga precisar/deseja realizar ou aquelas atividades que são esperadas que ele realizasse, mas que não tem conseguido ou não está satisfeito com seu desempenho. São escolhidas até cinco atividades consideradas prejudicadas pelo entrevistado nas áreas do autocuidado, trabalho e lazer. Em seguida, são mensurados de 1 a 10 o desempenho e a satisfação para com o desempenho em cada atividade. Entretanto no presente estudo, foi utilizada somente a primeira etapa da Medida, ou seja, a identificação das atividades prejudicadas (Bastos; Mancini; Pyló, 2010).

Os usuários foram convidados a participar do estudo durante seus tratamentos no CAPS AD. A abordagem dos mesmos pelos pesquisadores envolveu a explicação dos objetivos do estudo, esclarecimento de dúvidas, e a assinatura do Termo de Consentimento Livre e Esclarecido. As entrevistas foram realizadas de maneira individual, nos três turnos de funcionamento dos CAPS, em locais reservados disponíveis nos serviços, obedecendo aos preceitos éticos. O estudo foi submetido e aprovado pelo Comitê de Ética e Pesquisa com Seres Humanos do Centro de Ciências da Saúde da Universidade Federal de Pernambuco, sob o Número do Parecer: 2.613.712 (CAAE nº 50047615.3.0000.5208).

Os Softwares SPSS 13.0 (Statistical Package for the Social Sciences) para Windows e o Excel 2010 foram utilizados para a análise estatística dos dados. Todos os testes foram aplicados com $95 \%$ de confiança. Os resultados estão apresentados em forma de tabela com suas respectivas frequências absoluta e relativa. A verificação da existência de associação para as variáveis categóricas foi realizada através do Teste Exato de Fisher.

\section{Resultados}

Dos 99 usuários classificados como elegíveis para a pesquisa, 24 receberam alta por abandono, 19 não foram encontrados após três tentativas de localização nos seus respectivos horários de tratamento, 7 receberam alta do serviço por motivos diversos (melhora, mudança de endereço, questões administrativas) e 4 não aceitaram responder ao estudo. Assim, participaram da pesquisa um total de 45 usuários.

As principais variáveis sociodemográficas são apresentadas na Tabela 1.

Tabela 1 - Características sociodemográficas de usuários do CAPS AD, 2017.

\begin{tabular}{|c|c|c|}
\hline Variáveis & $\mathrm{N}=45$ & $\%$ \\
\hline \multicolumn{3}{|l|}{ Sexo } \\
\hline Feminino & 7 & 15,6 \\
\hline Masculino & 38 & 84,4 \\
\hline \multicolumn{3}{|l|}{ Faixa etária } \\
\hline 19 a 29 anos & 3 & 6,7 \\
\hline 30 a 39 anos & 10 & 22,2 \\
\hline 40 a 49 anos & 15 & 33,3 \\
\hline 50 a 59 anos & 10 & 22,2 \\
\hline 60 anos ou mais & 7 & 15,6 \\
\hline \multicolumn{3}{|l|}{ Naturalidade } \\
\hline Recife & 33 & 73,3 \\
\hline Outras localidades & 12 & 26,7 \\
\hline \multicolumn{3}{|l|}{ Escolaridade } \\
\hline Até 8 anos de escolaridade & 22 & 48,9 \\
\hline Até 11 anos de escolaridade & 17 & 37,8 \\
\hline$\geq 12$ anos de escolaridade & 6 & 13,3 \\
\hline \multicolumn{3}{|l|}{ Estado Civil } \\
\hline Solteiro (a) & 20 & 44,5 \\
\hline Casado (a)/União Estável & 14 & 31,1 \\
\hline Separado (a) ou Viúvo (a) & 11 & 24,4 \\
\hline \multicolumn{3}{|l|}{ Ocupação } \\
\hline Trabalhadores diversos & 18 & 40,0 \\
\hline Sem trabalho formal & 27 & 60,0 \\
\hline \multicolumn{3}{|l|}{ Renda em Salário Mínimo } \\
\hline Até um salário mínimo & 17 & 37,8 \\
\hline De 1 a 2 salários mínimos & 17 & 37,8 \\
\hline Mais de 2 salários mínimos & 2 & 4,4 \\
\hline
\end{tabular}


Fonte: Autores.

Dos 45 participantes do estudo a maioria era do sexo masculino, variando entre 19 a 65 anos, mas predominantemente na faixa etária acima dos 40 anos, natural da cidade do Recife e solteiro. Em relação à escolaridade, 48,9\% relataram ter estudado até a conclusão do ensino fundamental. No que diz respeito à atividade produtiva, 27 (60\%) entrevistados estavam sem trabalho formal no momento, porém $36(80 \%)$ relataram receber algum tipo de renda. A renda dessa população variou entre proveniente de salários, trabalhos informais, bolsas de programas sociais, ajuda de familiares, entre outros.

Sobre o histórico e perfil do uso e abuso das substâncias (Tabela 2) observou-se que 44,4\% negaram uso no dia que responderam a pesquisa, enquanto a mesma porcentagem alegou estar abstinente no mínimo uma semana do uso de álcool e/ou outras drogas.

Tabela 2 - Características do histórico e perfil do uso e abuso das substâncias de usuários do CAPS AD, 2017.

\begin{tabular}{|c|c|c|}
\hline Variáveis & $\mathrm{N}=45$ & $\%$ \\
\hline \multicolumn{3}{|l|}{ Uso no dia } \\
\hline Sim & 5 & 11,1 \\
\hline Não & 20 & 44,4 \\
\hline Está abstinente & 20 & 44,4 \\
\hline \multicolumn{3}{|l|}{ Tipo de Droga* } \\
\hline Álcool & 44 & 97,8 \\
\hline Maconha & 15 & 33,3 \\
\hline Crack & 12 & 26,7 \\
\hline Cocaína & 9 & 20,0 \\
\hline Cola & 2 & 4,4 \\
\hline Tabaco & 25 & 55,6 \\
\hline Outras Drogas & 1 & 2,2 \\
\hline \multicolumn{3}{|l|}{ Motivo para consumo } \\
\hline Estresse & 13 & 28,9 \\
\hline Tristeza/Solidão & 7 & 15,6 \\
\hline Vontade/Fissura & 16 & 35,6 \\
\hline Diversão/Socialização & 13 & 28,9 \\
\hline Outros motivos & 17 & 37,8 \\
\hline \multicolumn{3}{|l|}{ Percebe alguma alteração na cognição } \\
\hline Sim & 37 & 82,2 \\
\hline Não & 8 & 17,8 \\
\hline \multicolumn{3}{|l|}{ Tipo de alteração na cognição } \\
\hline Memória & 23 & 62,2 \\
\hline Regulação do comportamento & 7 & 18,9 \\
\hline Atenção & 5 & 13,5 \\
\hline Raciocínio lento & 4 & 10,8 \\
\hline \multicolumn{3}{|l|}{ O uso de drogas alterou sua rotina diária } \\
\hline Sim & 35 & $\mathbf{7 7 , 8}$ \\
\hline Não & 10 & 22,2 \\
\hline \multicolumn{3}{|c|}{ O uso de drogas alterou seu comportamento em algum local } \\
\hline Sim & 33 & 73,3 \\
\hline Não & 12 & 26,7 \\
\hline \multicolumn{3}{|c|}{ O consumo de drogas afetou seu relacionamento com parentes/amigos } \\
\hline Sim & 36 & 80,0 \\
\hline Não & 9 & 20,0 \\
\hline \multicolumn{3}{|c|}{ Considera o seu consumo prejudicial a si, ou familiares } \\
\hline Sim & 45 & 100,0 \\
\hline Não & 0 & 0,0 \\
\hline \multicolumn{3}{|l|}{ Sob efeito da droga, já cometeu algum ato infracional } \\
\hline Sim & 23 & 51,1 \\
\hline Não & 22 & 48,9 \\
\hline
\end{tabular}

*Foram identificados usuários de uma droga singular, assim como usuários de múltiplas drogas, por isso, os valores apresentados não representam uma escolha única. Fonte: Autores.

As substâncias mais frequentemente utilizadas pelos usuários foram o álcool com (97,8\%), seguido pelo tabaco $(55,6 \%)$, maconha $(33,3 \%)$, cocaína $(20 \%)$, crack $(12 \%)$, entre outras. 
Quanto à idade de início do consumo, $38(84,4 \%)$ participantes afirmaram ter começado o uso antes dos 20 anos, sendo apresentados às drogas em sua maioria $(62,2 \%)$ por amigos. Em relação à frequência do consumo, $66,7 \%$ dos participantes relataram que, quando em uso, utilizavam diariamente as substâncias e 33,3\% utilizavam semanalmente. Os principais motivos para o consumo foram vontade/fissura (35,6\%), seguido de estresse e diversão/socialização, com a mesma proporção de $28,9 \%$ cada.

Dos prejuízos decorrentes do uso, $80 \%$ afirmaram que o consumo afetou o relacionamento com parentes e amigos, todos entrevistados consideraram o uso prejudicial para si mesmo ou para seus familiares e $44,4 \%$ relataram ter algum outro problema de saúde, decorrente ou não do uso. A rotina de vida diária foi alterada para 77,8\% dos participantes, enquanto 73,3\% apresentaram algum tipo de alteração de comportamento, 51,1\% relataram ter cometido algum ato infracional sob o efeito de substâncias psicoativas e 82,2\% perceberam algum tipo de alteração cognitiva, sendo a memória (62,2\%) e a regulação do comportamento $(18,9 \%)$ as funções mais prejudicadas, segundo os mesmos.

Em relação ao perfil cognitivo, como é apresentado na Tabela 3, 39 entrevistados tiveram o ponto de escolaridade adicionado aos seus escores finais, assim como 42 dos 45 participantes tiveram um escore indicativo de comprometimento cognitivo leve. A média do escore total dos participantes foi de 18 pontos. A média de pontuação de acordo com a faixa etária foi de: 19 a 29 anos (20 pontos); 30 a 39 anos (20 pontos); 40 a 49 anos (17 pontos); 50 a 59 anos (16 pontos); e acima dos 60 anos (17 pontos).

Tabela 3 - Características do perfil cognitivo dos usuários do CAPS AD, 2017.

\begin{tabular}{lcc}
\multicolumn{1}{c}{ Variáveis } & $\mathbf{N}=\mathbf{4 5}$ & $\mathbf{\%}$ \\
\hline Ponto Escolaridade & 6 & 13,3 \\
Não adicionou (>12 anos escolaridade) & 39 & 86,7 \\
Adicionou ( $\leq 12$ anos escolaridade) & & \\
MoCA & 42 & 93,3 \\
Comprometimento cognitivo leve & 3 & 6,7 \\
Sem comprometimentos cognitivos & \\
\hline
\end{tabular}

Fonte: Autores.

A análise do resultado do MoCA revelou uma baixa média de acerto em oito itens: fluência verbal (28\%); repetição de sentenças (31\%); série de números (36,5\%); abstração (40\%); evocação tardia $(42,6 \%)$; trilha, relógio e cubo tridimensional $(43,4 \%)$. Tais itens avaliam principalmente a linguagem, função executiva, habilidade visuo-espacial e memória de curto prazo. Os itens que obtiveram as melhores médias de acerto foram: orientação (90,3\%); nomeação de animais (77,6\%); série de letras $(73 \%)$ e subtração $(58,3 \%)$. Os domínios cognitivos principalmente avaliados nesses itens são a atenção, concentração, memória de trabalho e orientação.

O estudo também se propôs a verificar o desempenho ocupacional desses usuários e nesse sentido a análise do COPM apontou até cinco atividades que cada participante julgava ter sido ou estar sendo prejudicada pelo uso das substâncias. No total, 33 atividades foram citadas como observa-se na Figura 1, de modo que as mais prevalentes foram: trabalhar $(35,77,8 \%)$, se relacionar com a família $(31,68,9 \%)$, estudar $(19,42,2 \%)$, cuidar das finanças $(18,40 \%)$ e cuidar da higiene pessoal $(15$, $33,3 \%$ ). A atividade de frequentar o CAPS foi apontada pelos entrevistados como prejudicada pelo uso, mas também como uma atividade de suporte social.

A análise buscou revelar a existência ou não de associação entre a variável dependente (alterações cognitivas) e as demais variáveis, de modo que a Tabela 4 mostra que houve associação estatisticamente significativa entre as variáveis escolaridade, frequência do uso de drogas, em relação ao comprometimento cognitivo. 
Figura 1 - Principais atividades com o desempenho comprometido de usuários do CAPS AD, 2017.

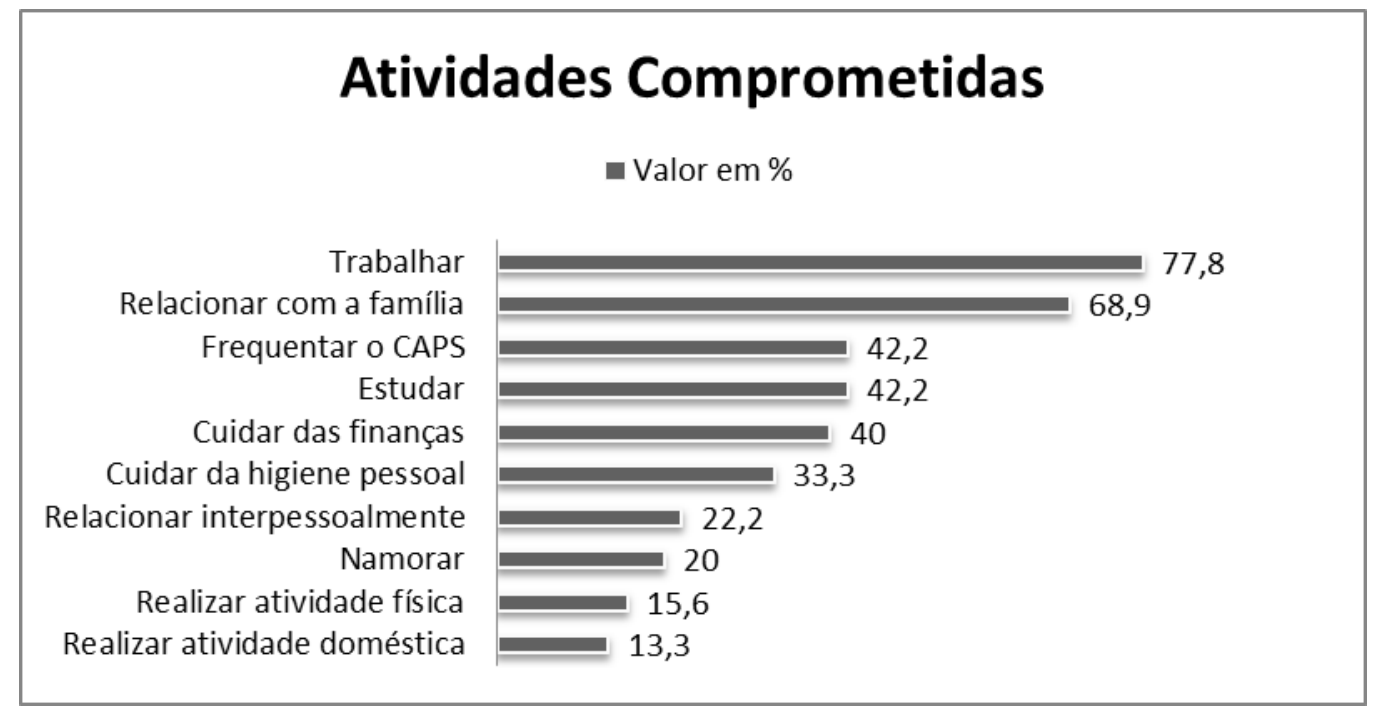

Fonte: Autores.

Tabela 4 - Associação entre Comprometimento Cognitivo, escolaridade, idade de início e frequência de uso das drogas, de usuários de CAPS AD, 2017.

\begin{tabular}{|c|c|c|c|}
\hline \multirow[b]{2}{*}{ Variáveis } & \multicolumn{2}{|c|}{ Comprometimento cognitivo } & \multirow[b]{2}{*}{ p-valor } \\
\hline & $\underset{\mathbf{n}(\%)}{\operatorname{Sim}}$ & $\begin{array}{c}\text { Não } \\
\text { n (\%) }\end{array}$ & \\
\hline \multicolumn{4}{|l|}{ Escolaridade } \\
\hline Até 8 anos de escolaridade & $21(95,5)$ & $1(4,5)$ & $0,043 *$ \\
\hline Até 11 anos de escolaridade & $17(100,0)$ & $0(0,0)$ & \\
\hline Igual ou maior que 12 anos de escolaridade & $4(66,7)$ & $2(33,3)$ & \\
\hline \multicolumn{4}{|l|}{ Idade de Inicio das Drogas } \\
\hline Até 20 anos & $35(92,1)$ & $3(7,9)$ & $1,000 *$ \\
\hline Mais de 20 anos & $7(100,0)$ & $0(0,0)$ & \\
\hline \multicolumn{4}{|l|}{ Frequência do uso de drogas } \\
\hline Diariamente & $30(100,0)$ & $0(0,0)$ & $\mathbf{0 , 0 3 2} *$ \\
\hline Semanalmente & $12(80,0)$ & $3(20,0)$ & \\
\hline
\end{tabular}

(*) Teste Exato de Fisher. Fonte: Autores.

\section{Discussão}

O número de participantes no estudo foi de 45 e pode sugerir um alto índice de abandono e baixa assiduidade ao tratamento, quando se compara ao número de sujeitos elegíveis para a pesquisa que eram 99. Essa situação corrobora com estudos anteriores que apontam diversos fatores possivelmente relacionados à adesão dos usuários de drogas ao tratamento, como a falta de apoio familiar, influência de amigos que também fazem uso da droga, não adequação ao tratamento, entre outros. O usuário pode não se adequar ao tratamento por diversas razões como não se sentir acolhido pelos profissionais (prejudicando o vínculo terapeuta-paciente), não reconhecer os benefícios do tratamento, não identificar as atividades realizadas como interessantes e significativas entre outras. Por esses motivos é importante a criação de estratégias de vinculação ao serviço, assim como o planejamento do projeto terapêutico individual focando no tratamento holístico, respeitando a história e singularidade de cada sujeito (Brasil, 2003; Galhardi \& Matsukura, 2018).

As principais caraterísticas da população do CAPS são homens, com idade acima dos 40 anos, porém com início do uso de drogas antes dos 20 anos, em concordância com diversos estudos, embora esses mesmos estudos ressaltem que a diferença de gênero vem diminuindo ao longo dos anos (Mastroianni, Macris, Gomes, \& Camargo, 2016; Santana, Miralles, Alves, dos Santos, Vinholes, \& da Silveira, 2020). Os achados no presente estudo são corroborados por estes autores, que 
obtiveram uma população prevalente acima dos 35 anos. Tais dados sugerem maior risco à população masculina na fase da adolescência, embora a faixa etária predominante em tratamento neste estudo seja de adultos, sugerindo que apesar do uso precoce é na fase adulta que o usuário passa a apresentar ou reconhecer os prejuízos causados pelas drogas.

Em seu estudo Mastroianni et al. (2016) averiguou grande parte de sua população com ensino fundamental incompleto, assemelhando-se ao resultado encontrado e ao relato dos participantes que apontaram estudar como atividade prejudicada pelo uso de drogas. Sabendo-se da baixa escolaridade em usuários de SPA e uso precoce na adolescência é possível inferir que o álcool e outras drogas podem ter efeito sob o baixo rendimento e abandono escolar, caracterizando-se como fator de risco e vulnerabilidade para essa população. Uma vez que se tem conhecimento da população e faixa etária de risco, é possível ter um norteamento de estratégias e políticas para crianças e adolescentes junto às escolas, visando a prevenção do primeiro uso e a prevenção da evasão escolar.

O álcool, o tabaco e a maconha foram apontados como as drogas mais consumidas, em consonância com estudos que as apontam como as drogas mais consumidas no Brasil (Laranjeira, Madruga, Pinsky, Caetano, Mitsuhiro, \& Castello, 2014; Santana et al., 2020). Dentre as demais categorias de drogas ilícitas foram mais prevalentes o crack, a cocaína, a cola (solvente) e medicamentos ansiolíticos (utilizados de forma não terapêutica), assemelhando-se com estudo de Almeida (2017) em CAPS AD da cidade do Recife. Porém, de acordo com a população que está sendo investigada pode haver diferença entre a prevalência das drogas. A identificação das drogas utilizadas pelos usuários dos serviços pode ajudar os profissionais no direcionamento das estratégias de intervenção, uma vez que cada droga tem sua ação no sistema nervoso, afetando de diferentes formas a percepção, humor, cognição e comportamento (Jurado Barba, Martínez, Sion, Álvarez Alonso, Robles, Quinto Guillén, \& Rubio, 2017; Fernández-Serrano, Pérez-García, \& Verdejo-García, 2011).

Notou-se que os participantes se mostraram conscientes dos prejuízos decorrentes do álcool e outras drogas. A maioria reconheceu o prejuízo a si e familiares, assim como no relacionamento com os mesmos, além de perceberem alterações em seus comportamentos e alterações cognitivas. Observou-se que durante a aplicação dos questionários os participantes refletiram sobre os prejuízos do uso das substâncias. A reflexão gerada através de momentos de conscientização pode ser utilizada como uma ferramenta de motivação para o tratamento.

Em relação à cognição, o comprometimento cognitivo leve foi identificado na maioria dos participantes. Através da análise do instrumento, foi possível observar menor desempenho nos itens de fluência verbal, repetição de sentenças, série de números, abstração, evocação tardia, trilha, relógio e cubo tridimensional. Entretanto, os itens não devem ser analisados individualmente, uma vez que os domínios cognitivos podem ser avaliados em mais de um item (Freitas, Simões, Alves, \& Santana, 2013). A análise dos itens descritos apontou maior prejuízo nos domínios da linguagem, função executiva, habilidade visuo-espacial e memória de curto prazo. Enquanto a maior parte dos participantes relatou perceber alteração na memória, regulação do comportamento (função executiva), atenção e raciocínio. Os achados são similares aos encontrados por Bernardin, Maheut-Bosser, e Paille (2014) que identificaram maior prejuízo na memória, habilidades viso-espaciais, atenção e linguagem.

Estudos apontam que o desempenho cognitivo atinge o seu pico pelos 30 anos, mas que começam a diminuir por volta dos 50 - 60 anos. No entanto, as perdas cognitivas não ocorrem da mesma forma em todas as pessoas idosas, pois além da idade, outras variáveis interferem no declínio cognitivo como nível educacional e condições socioeconômicas (Paulo \& Yassuda, 2010; Rosset, Roriz-Cruz, Santos, Haas, Fabrício-Wehbe, \& Rodrigues, 2011). Nesse estudo pôde-se observar o declínio cognitivo com o decorrer da idade, apesar dos participantes acima dos 60 anos terem tido um desempenho levemente superior aos de 50 a 59 anos. A diferença pode ser pelo tamanho da amostra de usuários acima dos 60 anos e pelas demais variáveis do histórico de vida. 
Maio e Caleiro (2011) utilizou o mesmo instrumento de avaliação cognitiva, com a mesma população de usuários de SPA e assim como no presente estudo a maioria dos participantes apresentaram comprometimento cognitivo. Entretanto, notase maior frequência de usuários com prejuízos cognitivos nos estudos brasileiros em comparação com estudos internacionais (Marceau, Lunn, Berry, Kelly, \& Solowij, 2016). Tal comparação levanta o questionamento sobre o perfil dos usuários de substâncias, bem como o sistema educacional e a qualidade de tratamento ofertado aos usuários nacionais e internacionais de drogas, como possibilidades que justificam a diferença encontrada no comprometimento cognitivo.

A associação entre o comprometimento cognitivo, a baixa escolaridade e a frequência diária do uso das substâncias revela uma magnitude importante acerca dos prejuízos que as drogas podem causar. Embora não se possa afirmar uma relação de causa e efeito, a associação entre a baixa escolaridade e o prejuízo cognitivo é discutida em diversos estudos (Freitas et al., 2013; Schilindwein-Zanini, R. Sotili, 2019; Cavalcanti et al, 2020). Contudo, é escasso na literatura trabalhos que estudem a relação entre a frequência do uso e os prejuízos que possam ocorrer ou não decorrente do uso frequente. Sugere-se que futuros estudos se aprofundem na análise desta variável.

Segundo a Estrutura da Prática da Terapia Ocupacional (2020), as ocupações são os vários tipos de atividades nas quais indivíduos, grupos ou populações se envolvem. As atividades observadas como prejudicadas pelos usuários são atividades de AVD, AIVD, educação, trabalho e participação social, demonstrando um prejuízo em diversas esferas da vida cotidiana.

Observou-se que, trabalhar, se relacionar com a família, estudar, namorar e se relacionar interpessoalmente são referidas como as atividades comprometidas pelo uso de drogas, corroborando com os resultados de Henrique, Silva e Pedrosa (2015) com a mesma população. O estudo com adolescentes do sexo do sexo feminino de Menezes e Pereira (2019) também reforçam os resultados observados, apontando um prejuízo no autocuidado, assim como no estudar, mostrando um comprometimento desde o início do uso ainda na adolescência.

Lima Júnior et al. (2016) estudou o comprometimento atencional entre trabalhadores dependentes químicos, e concluiu um pior desempenho destes trabalhadores comparado com os demais trabalhadores, apontando alterações cognitivas no domínio específico da atenção, indicando a possibilidade de limitações laborais, riscos de acidente de trabalho e diminuição qualidade de vida desses empregados. Tais informações corroboram com o alto número de usuários no atual estudo que referiram prejuízos nas atividades de trabalho.

O trabalho e a relação familiar aparecem predominantemente como atividades mais comprometidas. Considerando que a fase adulta é cercada pela expectativa social da produtividade no trabalho e da constituição familiar, compreende-se que sejam exatamente essas as atividades com um maior prejuízo no desempenho ocupacional, necessitando, portanto, de integração às estratégias de intervenção e de cuidado. O desempenho ocupacional satisfatório está relacionado não só às questões intrínsecas do sujeito mas também à sua relação com o ambiente e com a promoção de saúde e bem-estar (Pereira et al, 2014).

As habilidades cognitivas podem estar alteradas nos usuários de substâncias psicoativas afetando o desempenho ocupacional dos mesmos, visto que a cognição influencia nos processos de volição, escolha, organização e execução de atividades (Ferreira \& Colognese, 2014). Carvalho; Oliveira; Pinto (2021) também compartilham em seu estudo que as funções cognitivas de usuários que fazem uso de substâncias psicoativas são afetadas e que esses prejuízos podem ser trabalhados nos serviços de saúde mental, objetivando a melhora do estado de saúde, funcionalidade e qualidade de vida do indivíduo.

A AOTA (2020) traz que o nível cognitivo aparece como um componente essencial para as demandas de atividades e ocupações. Assim como as funções anteriormente sinalizadas como prejudicadas aparecem como fatores intrínsecos do sujeito que influenciam o desempenho do mesmo em ocupações. Identificar os fatores que estão comprometidos é de grande 
importância para o planejamento dos objetivos e metas do tratamento, assim é possível potencializar as funções preservadas e estimular as funções prejudicadas.

\section{Conclusão}

O estudo verificou que o uso de substâncias psicoativas tem acontecido precocemente, principalmente na população masculina, com baixo nível de escolaridade. Além disso, observou-se associação entre o comprometimento cognitivo, a baixa escolaridade e a frequência do uso de drogas. Os usuários do CAPS AD apresentaram comprometimento cognitivo leve e os domínios mais prejudicados foram: linguagem, habilidade visuoespacial, memória de curto prazo e função executiva. Observou-se uma grande variedade de atividades referidas como prejudicadas pelos participantes, todavia trabalhar e se relacionar com familiares foram as mais citadas entre a maioria.

Diante dos achados ressalta-se a importância de políticas públicas voltadas à prevenção junto à população de risco. Considera-se importante a identificação das funções prejudicadas e avaliação do impacto destas nas diversas atividades cotidianas. Esses aspectos podem facilitar o direcionamento do tratamento, visando a melhora do desempenho ocupacional, consequentemente a melhora da qualidade de vida e a facilitação da reinserção social. Recomenda-se que haja sensibilidade dos profissionais, principalmente da Terapia Ocupacional, em direcionar os usuários a atividades significativas, que possam operar no processo da reabilitação cognitiva, voltada para as ocupações, assim como na aquisição de novas habilidades.

Vale ressaltar a aplicabilidade do instrumento de avaliação cognitiva MoCA com usuários de álcool e outras drogas. A rápida aplicação, boa precisão e sensibilidade em identificar comprometimento cognitivo o tornam indicado à prática clínica.

No decorrer da pesquisa, algumas limitações foram encontradas, como o tamanho da amostra e a inviabilidade da coleta de dados com usuários de todas as modalidades de tratamento do CAPS. Sugere-se estudos futuros com todos os usuários dos serviços, a fim de um retrato mais amplo da população.

\section{Agradecimentos}

A todos os coordenadores e trabalhadores dos CAPS pela disponibilidade e empenho. Aos usuários que dedicaram seu tempo e doaram um pouco de suas histórias. Às colegas, Tayná Araújo e Elba Amanda, agradeço pela companhia e ajuda no processo da coleta de dados.

\section{Referências}

Almeida, N. D. (2017). Uso de álcool, tabaco e drogas por jovens e adultos da cidade de Recife. Psicologia argumento, 29(66).

American Occupational Therapy Association - AOTA. (2013). Cognition, Cognitive Rehabilitation, and Occupational Performance. American Journal of Occupational Therapy, 67.

American Occupational Therapy Association - AOTA. (2020). Occupational Therapy Practice Framework: Domain and Process Fourth Edition. (4a ed.), The American Journal of Occupational Therapy, 74(2).

Andrade, L. S. et al. (2018). Ritalina uma droga que ameaça a inteligência. Rev Med Saude Brasilia. 7(1):99-112.

Assunção, J. I. V. et al. (2019). Eiras E Beiras: Atenção Psicossocial A Pessoas Com Problemas Decorrentes Do Uso De Drogas. Psicologia \& Sociedade, 31 : e178671.

Bastos, S. C. D. A., Mancini, M. C., \& Pyló, R. M. (2010). O uso da Medida Canadense de Desempenho Ocupacional (COPM) em saúde mental. Revista de Terapia Ocupacional da Universidade de São Paulo, 21(2):104-110.

Bernardin, F., Maheut-Bosser, A., \& Paille, F. (2014). Prejuízos cognitivos em dependentes de álcool. Fronteiras em psiquiatria, 5 , 78.

Brasil. A política do Ministério da Saúde para a atenção integral a usuários de álcool e outras drogas. <http://bvsms.saude.gov.br/bvs/pu blicacoes/politica_atencao_alcool_drogas.pdf $>$. 
Brasil. Ministério da Saúde, Gabinete do Ministro, Portaria N $N^{o} .088$, de 23 de dezembro de 2011 Institui a Rede de Atenção Psicossocial para pessoas com sofrimento ou transtorno mental e com necessidades decorrentes do uso de crack, álcool e outras drogas, no âmbito do Sistema Único de Saúde (SUS), 2011. <http://bvsms.saude.gov.br/bvs/saudelegis/gm/2011/prt3088_23_12_2011_rep.html>.

Carvalho, J. P. S. S. Oliveira, C. G. A. \& Pinto, C. A. P. (2021). O uso de substâncias psicoativas e o estado mental: avaliando a função cognitiva através do Mini Exame do Estado Mental. Revista Eletrônica Acervo Saúde, 13(2):1-7.

Cavalcanti, A. D. C. et al. (2020) Alterações Neurofisiológicas E Cognitivas Decorrentes Do Uso Crônico Da Maconha: Uma Revisão De Literatura. Ciências Humanas e Sociais, 6(1): 85-92.

Ferreira, V. R. T., \& Colognese, B. T. (2014). Prejuízos de funções executivas em usuários de cocaína e crack: case studies. Avaliação Psicológica: Interamerican Journal of Psychological Assessment, 13(2), 195-201.

Fernández-Serrano, M. J., Pérez-García, M., \& Verdejo-García, A. (2011). What are the specific vs. generalized effects of drugs of abuse on neuropsychological performance? Neuroscience and Biobehavioral Reviews, 35(3): 377-406.

Freitas, S., Simões, MR, Alves, L., \& Santana, I. (2013). Avaliação cognitiva de Montreal: estudo de validação para comprometimento cognitivo leve e doença de Alzheimer. Alzheimer Disease \& Associated Disorders , 27 (1): 37-43.

Galhardi, C. C., \& Matsukura, T. S. (2018). O cotidiano de adolescentes em um Centro de Atenção Psicossocial de Álcool e outras Drogas: realidades e desafios. Cadernos de Saúde Pública, 34, e00150816.

Guariento, C. F. (2019). Prevenção e promoção de saúde no CAPS AD através de oficinas de psicoeducação. Rev. Elet. Cient. da UERGS, . 5(especial):191197.

Henrique, A. R. P., Silva, T. F., \& Pedrosa, É. M. (2015). Comprometimentos Do Desempenho Ocupacional De Toxicômanos. Revista Científica Linkania Master, 1(10).

Jafelice, G.T. Ziliotto, G.C. Marcolan, J.F. (2020). Concepção dos profissionais de centro de atenção psicossocial sobre as politicas públicas de saúde mental. Research, Society and Development, 9(5): e71953100.

Jurado Barba, R., Martínez, A., Sion, A., Álvarez Alonso, M. J., Robles, A., Quinto Guillén, R., \& Rubio, G. (2017). Development of a screening test for cognitive impairment in alcoholic population: TEDCA.

Laranjeira, R. et al. (Org.). II Levantamento Nacional de Álcool e Drogas (LENAD) - 2012Instituto Nacional de Ciência e Tecnologia para Políticas Públicas de Álcool e Outras Drogas (INPAD). São Paulo: Instituto Nacional de Ciência e Tecnologia para Políticas Públicas de Álcool e Outras Drogas (INPAD), UNIFESP, 2014. <http://inpad.org.br/wp-content/uploads/2014/03/Lenad-II-Relat\%C3\%B3rio.pdf>.

Laranjeira, R., Madruga, C. S., Pinsky, I., Caetano, R., Mitsuhiro, S. S., \& Castello, G. (2014). II levantamento nacional de álcool e drogas (LENAD)-2012. São Paulo: Instituto Nacional de Ciência e Tecnologia para Políticas Públicas de Álcool e Outras Drogas (INPAD), UNIFESP.

Lima Júnior, O. A. et al. (2016). Os impactos na capacidade atencional em trabalhadores usuários de drogas. Revista Brasileira de Medicina do Trabalho, 14 (2): 84-8.

Machado, A. P., et al. (2021). Perfil dos usuários cadastrados em um Centro de Atenção Psicossocial - Álcool e Drogas (CAPS-AD). Brazilian Journal of Health Review, 4(2) 7603-7609.

Maio, T. P., \& Caleiro, F. M. (2011). Aplicabilidade do instrumento montreal cognitive assessment para dependentes químicos de duas cidades da região sul do rio grande do sul. Artigo Parcial, 1(2).

Marceau, E. M., Lunn, J., Berry, J., Kelly, P. J., \& Solowij, N. (2016). The Montreal Cognitive Assessment (MoCA) is sensitive to head injury and cognitive impairment in a residential alcohol and other drug therapeutic community. Journal of substance abuse treatment, 66, 30-36.

Mastroianni, F. C., Macris, C. E., Gomes, J. R., \& Camargo, P. J. (2016). Perfil sociodemográfico de um CAPSad e sua funcionalidade segundo os usuários. Rev. Psicol. Saúde, 8(2): 3-16.

Memória, C. M. et al. (2013) Brief screening for mild cognitive impairment: Validation of the Brazilian version of the Montreal cognitive assessment. International Journal of Geriatric Psychiatry, Chichester, 28(1): 34-40.

Menezes, A. L. \& Pereira, A.R. (2019). Desempenho ocupacional de adolescentes usuárias de drogas. Cad. Bras. Ter. Ocup, 27(4): 754-764.

Moraes, P. L. M. et al (2020). A percepção da desinstitucionalização pelos profissionais da saúde mental na práxis no Centro de Atenção Psicossocial no contexto brasileiro. Research, Society and Development, 9(7): e763974750.

Nasreddine, Z. S. et al. (2005) The Montreal Cognitive Assessment, MoCA: a brief screening tool for mild cognitive impairment. Journal of the American Geriatrics Society, 53(4):695-699.

Oliveira, L. C., Mascarenhas, C. H. M., \& Melo, N. S. A. (2014). Qualidade de vida e independência funcional de usuários de drogas atendidos em um Centro de Atenção Psicossocial Álcool e Drogas (CAPSad). Rev Bras Qual Vida [Internet], 6(4), 232-40.

Paulo, D. L. V., \& Yassuda, M. S. (2010). Queixas de memória de idosos e sua relação com escolaridade, desempenho cognitivo e sintomas de depressão e ansiedade. Archives of Clinical Psychiatry, 37(1), 23-26.

Pedrero-Pérez, E. J. et al. (2014). Reserva cognitiva en adictos a sustancias en tratamiento: Relación con el rendimiento cognitivo y las actividades cotidianas. Revista de Neurologia, 59(11): 481-489. 
Research, Society and Development, v. 10, n. 10, e223101018483, 2021

(CC BY 4.0) | ISSN 2525-3409 | DOI: http://dx.doi.org/10.33448/rsd-v10i10.18483

Pelletier, S. et al. (2016) Investigation of Cognitive Improvement in Alcohol-Dependent Inpatients Using the Montreal Cognitive Assessment (MoCA) Score. Journal of Addiction, 2016: 1-7.

Pereira, D. C., et al. (2014). Desempenho ocupacional de adolescentes. Rev Ter Ocup Univ São Paulo, 25(1):11-17.

Rosset, I., Roriz-Cruz, M., Santos, J. L. F., Haas, V. J., Fabrício-Wehbe, S. C., \& Rodrigues, R. A. (2011). Socioeconomic and health differentials between two community-dwelling oldest-old groups. Revista de saude publica, 45, 391-400.

Santana, R. T., Miralles, N. C. W., Alves, J. F., dos Santos, V. Á., Vinholes, U., \& da Silveira, D. S. (2020). Perfil dos usuários de CAPS-AD III. Brazilian Journal of Health Review, 3(1), 1343-1357.

Henrique, A. R. P., Silva, T. F., \& Pedrosa, É. M. (2015). Comprometimentos do desempenho ocupacional de toxicômanos. Revista Científica Linkania Master, 1: 95-115.

Schilindwein-Zanini, R. \& Sotili, M. (2019). Uso De Drogas, Repercussões E Intervenções Neuropsicológicas. Cadernos Brasileiros de Saúde Mental, 11(28):94-116.

Silva, M. A. B., \& Abrahão, A. L. (2019). Política de Atenção Integral aos usuários de álcool e outras drogas: uma análise guiada por narrativas. InterfaceComunicação, Saúde, Educação, 24.

Sistema para detecção do uso abusivo e dependência de substâncias psicoativas: encaminhamento, intervenção breve, reinserção social e acompanhamento SUPERA. (2017). Deteç̧ão do uso e diagnóstico da dependência de substâncias psicoativas: módulo 3.

Vargas, A. F. M. \& Campos, M. M. (2019). A trajetória das políticas de saúde mental e de álcool e outras drogas no século XX. Ciência \& Saúde Coletiva, 24(3):1041-1050.

World Health Organization - WHO. Global status report on alcohol and health 2014Global status report on alcohol. Geneva, 2014. <http://www.who.int/substance_abuse/publications/global_alcohol_report/msbgsruprofiles.pdf >.

World Health Organization - WHO. (2018). Global status report on alcohol and health 2018 Global status report on alcohol. <file:///C:/Users/Tom\%20Produ\%C3\%A7\%C3\%B5es\%2008/Downloads/9789241565639-eng.pdf> 